\title{
Framework of Autonomous Damage of Hazards Detection with Rescue Route Identification For UAV Implementing IoT and DIP
}

\author{
Mohadmmad Amzad Hossain ${ }^{1}$, Mohammad Tashauf Akand ${ }^{2}$, Iftikharul Faridee Nur ${ }^{3}$, Sazzatuz Zumma Ismam ${ }^{4}$, \\ MD. Fahim Shahriar Sakib ${ }^{5}$, Tanvir Ahmed ${ }^{6}$, Azmir Ahmed ${ }^{7}$ \\ ${ }_{1,3}^{3}$ Department of Computer Science and Engineering, BRAC University \\ ${ }^{2}$ Department of Computer Science and Engineering, American International University Bangladesh \\ ${ }^{4}$ Department of Electronics and Communications Engineering, East West University \\ ${ }^{5,7}$ Department of Electrical and Electronic Engineering, BRAC University \\ ${ }^{6}$ Department of Electrical and Electronic Engineering, American International University Bangladesh \\ Correspondence Author - Sazzatuz Zumma Ismam, sazzatuzismam92@gmail.com
}

DOI: 10.29322/IJSRP.11.02.2021.p11051

http://dx.doi.org/10.29322/IJSRP.11.02.2021.p11051

\begin{abstract}
Numerous hazards that are undermine the world, can cause loss of lives or damage and all of them have the ability to cause severe damage to homes, organizations and framework. These incorporates meteorological risks, inadvertent dangers flooding, earthquake, volcano and so on. It has turned into a serious issue of concern all through the world, regardless of whether it is natural hazard or by human components. In this research work, we propose a hazard or disaster detection systems implementing image processing algorithms like holistically-nested edge detection, k-mean cluster, speeded up robust features algorithm. The whole theme of the work is divided into two secession. In the first secession, we present an algorithm for searching best route in an urban area analyzing before and after images of nature hazard from satellite. In the second phase, to automatically detect the affected areas of hazard or disaster and estimate the destruction before sending rescue teams or aids. The system is able to detect affected regions of fire and volcanoes, measure the flood affected zone and finally can estimate the damage occurred by human war destruction. Thus, this system is very essential for autonomous natural and human detection.
\end{abstract}

Index Terms- Hazard damage detection, image processing, fastest route selection, holistically-nested edge detection, k-mean cluster, speeded up robust features algorithm.

\section{INTRODUCTION}

$\mathrm{N}$ atural disasters is one of the core reasons of economical struggle of any country. It causes huge global economical drawback which takes lots of time to be covered. In this modern era, fast post disaster management is still a great challenge for aid supplement, speciallay for the developing or undeveloped countries. Government has to spend a lots of money just to get idea of the distruction caused by the hazards. Moreover, natural hazards like earthquakes in city may create a route selecting problems. As a result, rescuing people in short of time as well as sending necessary goods can't be possible. However, scientist is proposing satellite based solution which is very costly and has to depend to the controlling countries; additionally it takes time to be processed. In our research work, we've proposed to developed a camera based low cost quad copter with auto flying technique that will take offline map's location coordinates combining with longitude and latitude. The quad copter works as an area observing satellite and the camera captures several ground images which are gone through image processing techniques and our proposed route selecting algorithm to measure the destruction and figure out the shortest route. The rest of this paper is organized as follows: Section 2contains the literature reviews where related works of other papers have seen showed. Section II provides a briefly discuss the proposed methodology in experimental setup division. Section III is the results and demonstration part which are obtained the methodology. In the end, section IV concludes the paper combining with future works.

A. J. Cooner et al [2] demonstrated the post disaster analysis implementing machine learning approaches on images of earthquakes of Haiti of 2010 using texture and structural statistical features like entropy, Palladian of Gaussian, dissimilarity, and rectangular fit. For classifying based on the features multilayer feed forward neural nets, Random Forest classifiers and radial basis neural nets were applied to compare their result where each of algorithms was able to scores almost $90 \%$ kernel density match. The authors finally claimed in the end that textural and structural spatial features were more significant in improving the accuracy of the research work.

$\mathrm{S}$. W. Myint et al[1] proposed remote sensing and image processing methods to identify tornado damaged zones. The authors showed a comparison of principal component investigation, Image differencing along with object-oriented classification. They demonstrated that the damaged and undamaged regions have contrasts in the spectral reflectance. Their proposed object-oriented classification was able to attain better accuracy than the other two mentioned strategies. 
A. Sumalan et al [3] showed detection of flooded zone using an unsupervised technique implementing images captured by a fixedwing unmanned aerial vehicle. Despite their theory for the detection of the flooded areas containing with an algorithm based on kmeans clustering and texture analysis using co-occurrence matrix, the authors emphases on UAV images where the system that applies images increase the usability by common people.

\section{EXPERIMENTAL SETUP}

The proposed works are mainly divided into three major sections. Firstly, we discussed about the autopilot system of a quad copter based on calculating longitude and latitude, then secondly total affected areas of natural hazard are detected and finally shortest route for rescue is computed. Figure 01 shows the workflow of this proposed methodology.

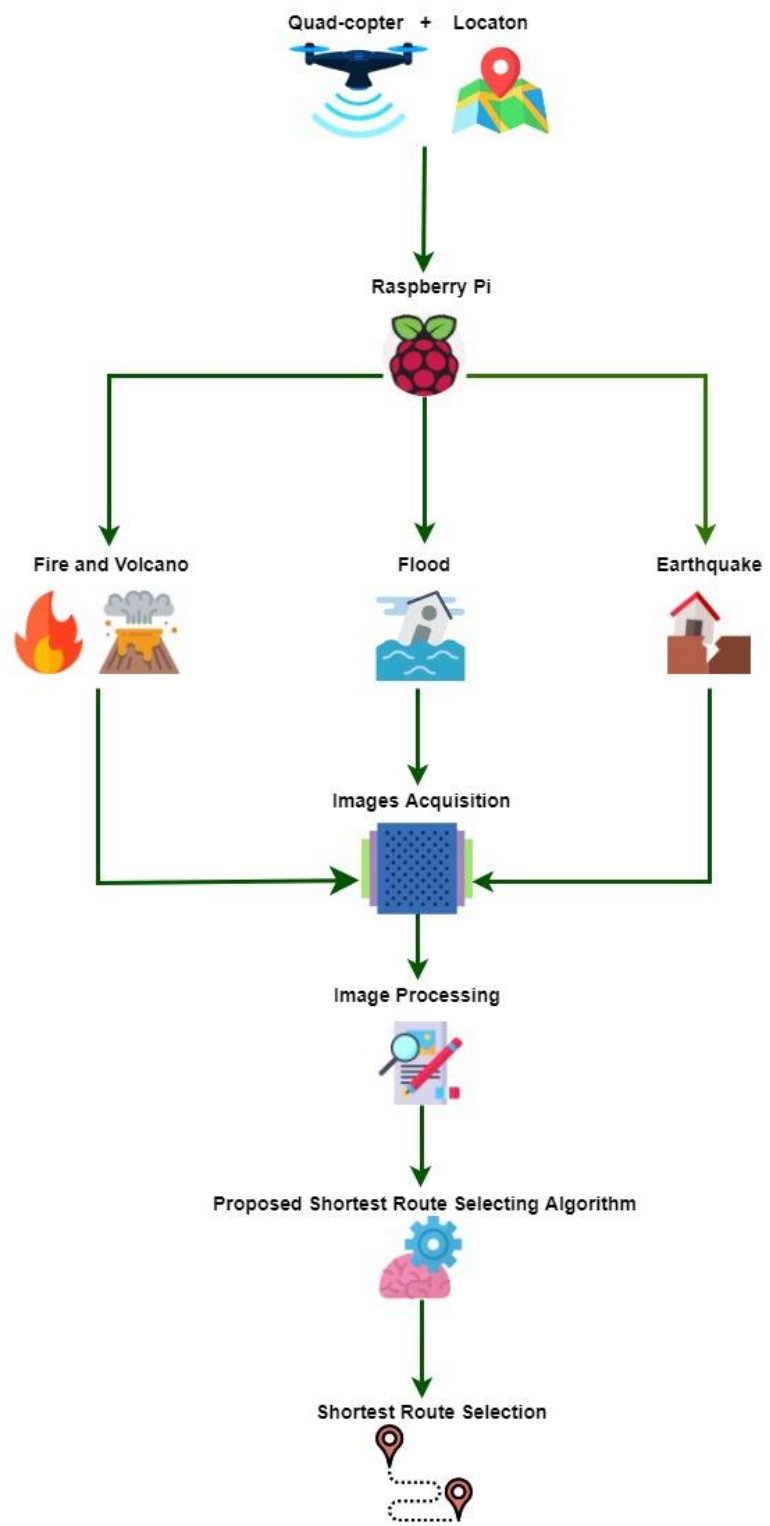

Figure 1. Proposed work diagram

A. Self-Driven Quad copter concept and Edge Computing: To get and analysis satellite or aerial images using quad copter, it might be very harder to control and communicating part as it has to go a long way and bad weather conditions affects the communication. So, we proposed an autopilot system for quad copter to go to the specific place based on the longitude and latitude from offline map. The proposed system calculates its distance based on the current and destinations longitude and latitude using sine formula, as well as degree of turning fetching data from offline map. 
B. Image processing for hazard's damage detection: Image processing techniques are applied to segment and detected hazard affected part and calculate the total area of distraction to take further steps. To process the images, it needs to be convert into CIE $\mathrm{L} * \mathrm{a} * \mathrm{~b} *$ color space [4]. The conversion of RGB to CIE L*a*b* color space [4] is formulated as follows:

$$
\begin{aligned}
& {\left[\begin{array}{l}
P \\
Q \\
R
\end{array}\right]=\left[\begin{array}{ccc}
0.412453 & 0.357580 & 0.180423 \\
0.212671 & 0.715160 & 0.072169 \\
0.019334 & 0.119793 & 0.950227
\end{array}\right]\left[\begin{array}{l}
R \\
G \\
B
\end{array}\right]} \\
& L *=116 * f\left(\frac{Q}{Q_{n}}\right)-16 \\
& a *=500\left[f\left(\frac{P}{P_{n}}\right)-f\left(\frac{Q}{Q_{n}}\right)\right] \\
& b *=200\left[f\left(\frac{Q}{Q_{n}}\right)-f\left(\frac{R}{R_{n}}\right)\right]
\end{aligned}
$$

Here, P, Q, R, Pan, An, and Rn are the coordinates of CIEPQR colour space. The solution to convert digital images from the RGB space to the CIEPQR colour space is as the following formula. The data range of color channels is between 0 and 255 for 8 -bit data representation. The data ranges of $\mathrm{L}^{*}, \mathrm{a}^{*}$, and $\mathrm{b}^{*}$ components are $(0,100),(-110,110)$, and $(-110,110)$, respectively. The numeric color responses $\mathrm{L}^{*}, \mathrm{a}^{*}$, and $\mathrm{b}^{*}$ are normalized .It is assumed that the fire in an image has the brightest image region and is near to the color red. Where $\mathrm{L}^{*}, \mathrm{a}^{*}$, and $\mathrm{b}^{*}$ channels are quantized into 24 levels, and 6,223,467 pixels are used to create each histogram. The number of quantization levels can be changed, but through experimentation, 24 levels were found to give satisfactory results. A lookup table is created for each pair of 24 quantized levels to keep track of the likelihood that any pair of $\mathrm{L}^{*}$, $\mathrm{a}^{*}$, and $\mathrm{b}^{*}$ belongs to a fire. It is clear from Figs. 4(a), 4(b), and 4(c) that a fire can be defined by the combination of three histograms. Given the $\mathrm{L}^{*}$, $\mathrm{a}^{*}$, and $\mathrm{b}^{*}$ color values at spatial location $(x, y)$, the likelihood that $\mathrm{L}^{*}, \mathrm{a}^{*}$, and $\mathrm{b}^{*}$ belong to a fire $\mathrm{P}\left(\mathrm{L}^{*}, \mathrm{a}^{*}, \mathrm{~b}^{*}\right)$ is defined as where $\mathrm{P}\left(\mathrm{L}^{*}, \mathrm{a}^{*}\right)$.

The following converted image then processed using clustering algorithm named k-mean cluster (shown in Fingure 2.)which segment the color channels in k numbers. K-means algorithm [5-9], [4] is an iterative algorithm that tries to partition the dataset into $K$ predefined distinct non-overlapping clusters where each data point belongs to only one group. It tries to make the inter-cluster data points as similar as possible while also keeping the clusters as different as possible. It assigns data points to a cluster such that the sum of the squared distance between the data points and the cluster's centroid arithmetic mean of all the data points that belong to that is at the minimum. The less variation we have within clusters, the more homogeneous the data points are within the same cluster.

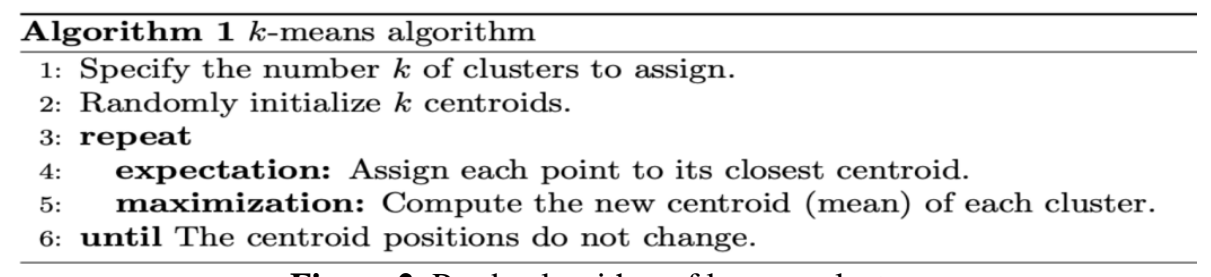

Figure 2. Psudo algorithm of k-mean cluster.

To do the analysis, we input two images, one is affected and another one is unaffected. To reduce the color complexity, we applied gray scaling and Gaussian blurring in this image, as a result low pass noise will be reduced. It also helps to reduce the [15],[16],[17] complexness not to detect the destroyed parts, roof of the house or region beside road. After applying adaptive thresholding, we convert the images binary image into and perform flood fill method to fill gaps [16]. Now, by the method of connected components, we measure the longest connected neighbor. The longest connected components will mark as road. Further change the pixel values and color the paths. The binary we got will be used to detect roads using $\mathrm{K}$ means clustering algorithm. Which will detect all the Euclidean points. Basically, $\mathrm{K}$ means clustering method will find the presence and absence of any object on that path and detect the road that are connected. Now we will test affected area after disaster with the same process. We will apply median filter, Gaussian Smoothing Filter and we will find the clear RGB image of road map. K means clustering will be applied to this image to detect the available routes. K means will try to find the presence and absence of an object and it finds an object on that path it will not count that.

C. Shortest Path Finding: While segmentation is done, it converted into binary image using binarization technique of adaptive thresholding. The proposed algorithm then scans from top bottom and left to right. From the point the value of y axes increases to any direction, it is stacked with its distance from the its straight image border and pixel distance value. Figure 3 shows the segmented road map after converting into masked binary image and marking major points [21]. 

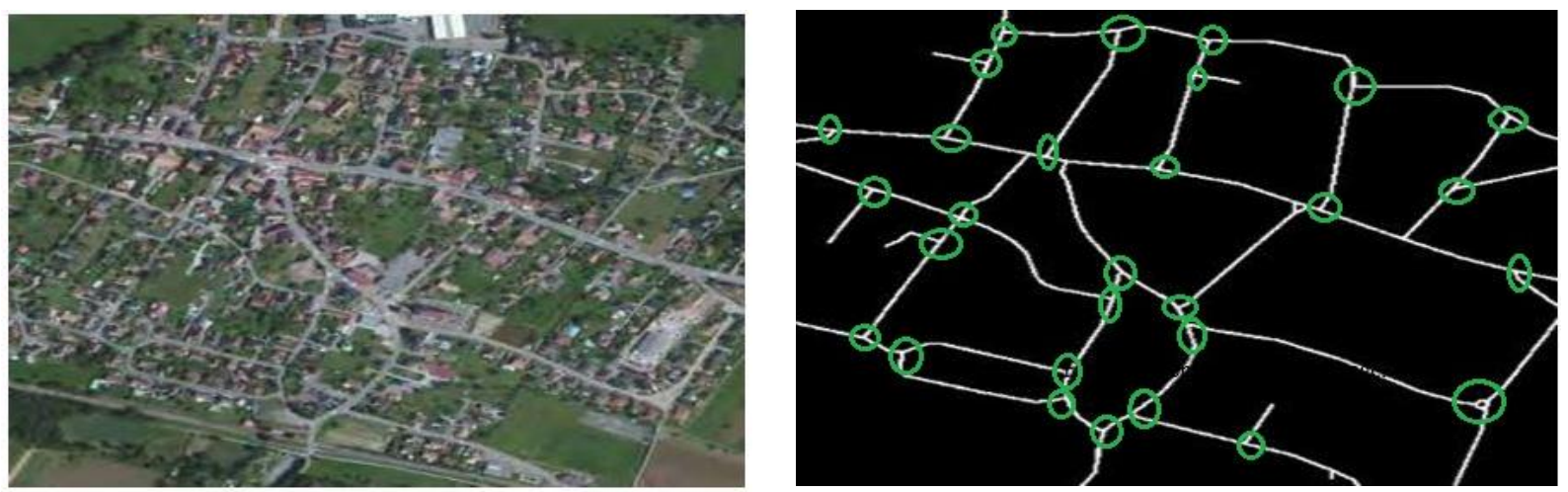

Figure 3. Inputted image and major point selection

Shortest path algorithm is considered as one of the suitable methods for searching best path.. we used a discrete directed graph as $\mathrm{G}$ $(\mathrm{V}, \mathrm{E})$. The set of vertex $\mathrm{V}=\{\mid \mathrm{i}=\mathrm{i}, \ldots, \mathrm{n}\}$ is defined as the set of intersections and ending points of a road. The set of edges $\mathrm{E}=\{$ $\mid \mathrm{i}=\mathrm{i}, \ldots, \mathrm{n}\}$ is defined as the set of road segments between a pair of adjacent intersections or ending points. There is a source position $\mathrm{s}$ and destination position $\mathrm{d}$. The main purpose of this algorithm is to find the shortest road from source to destination assuming that there is no obstacle on the road. Along with Dijkstra with heuristic based on the greedy best first search (BFS) is used to estimate the shortest path on the huge area of the map [10]. As there are some complexities in using heuristic searching technique.

\section{D. internet of Things using Raspberry $\mathbf{P i}$}

We use raspberry pi zero micro controller that has in built wi-fi module and personalized operating system which is very efficient for tasks like IoT [11],[12],[13], [14]. The photos saved from the Camera modules cannot be sent to the server directly, since the use of bandwidth is inefficient. Via base64 encoding technique, the images are first coded in a string. Base64 is a category of identical binary to text encoding schemes, which converts binary data into a radix-64 representation of an ASCII string format. This means that a lot of bandwidth is not used in the image. Based on different variables the savings ratio will be over 200. It is much easier to send images to the cloud since they now can be directly transmitted as POST data, instead of downloading a file. The Pi will keep sending the server updates at a predefined interval concerning the current position [18]. The update would include the UID, the location and the timestamps of the unit. The update is rendered with the aid of a python script using a POST request. After the predefined time, the script will read stored position data and other parameters and render a POST payload out, and the request will be shot. It repeats this step on a frequent basis.

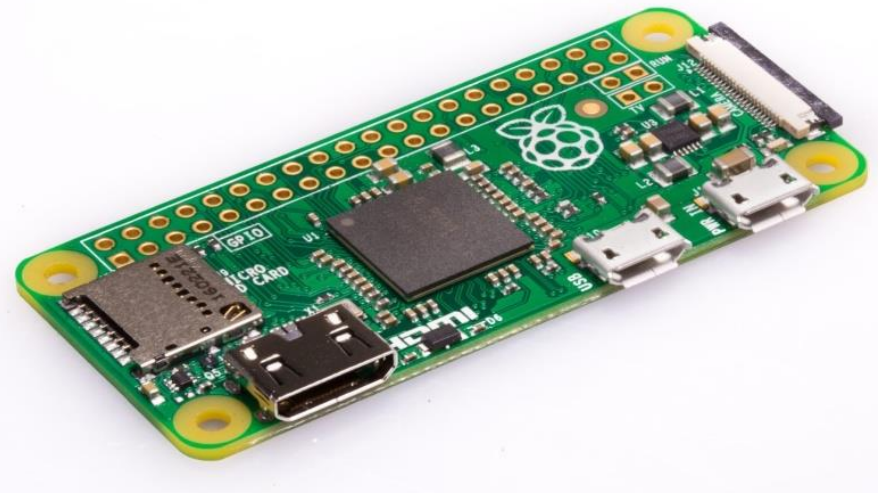

(a)

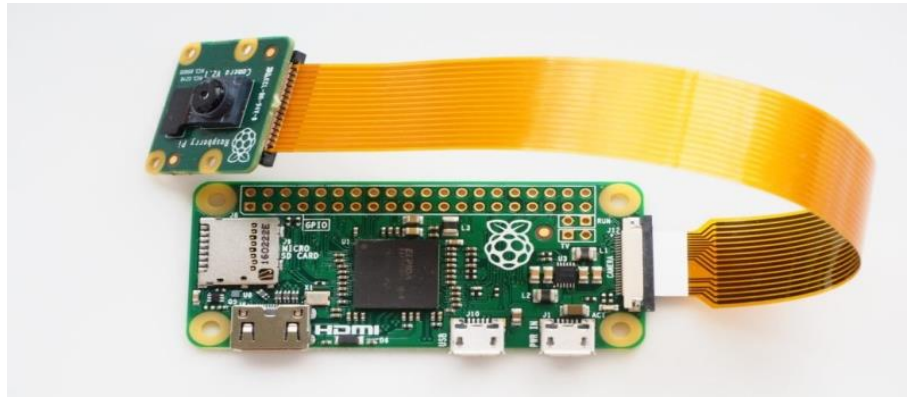

(b)

Figure 4. (a) Raspberry Pi Zero, (b) with camera module.

\section{RESULTS AND FINDINGS}

By using our proposed method, we got post disaster road map satellite images from different areas and also fire or volcano affected areas. Finally, we run a shortest path algorithm to find the shortest route to the affected are and this process happens in 3 stages described in Table 1. 
Table 1. Stages of shortest path algorithm

\begin{tabular}{|l|l|}
\hline Stage 01 & $\begin{array}{l}\text { By using Image processing techniques, we detect the normal roads of that area. This area map is previously stored in } \\
\text { database. }\end{array}$ \\
\hline Stage 02 & $\begin{array}{l}\text { Using the same technique comparing after disaster image and stored image we got newly detected roads on post } \\
\text { disaster image. }\end{array}$ \\
\hline Stage 03 & $\begin{array}{l}\text { Finally, we implemented the shortest path estimation algorithm to find the shortest road so that the relief team can } \\
\text { reach the destination as soon as possible. }\end{array}$ \\
\hline
\end{tabular}

The main motivation is to detect the road and marked theme on the basis of before after disaster, segmenting the vocation's part, finding the shortest routes and analyzing before after disaster images.

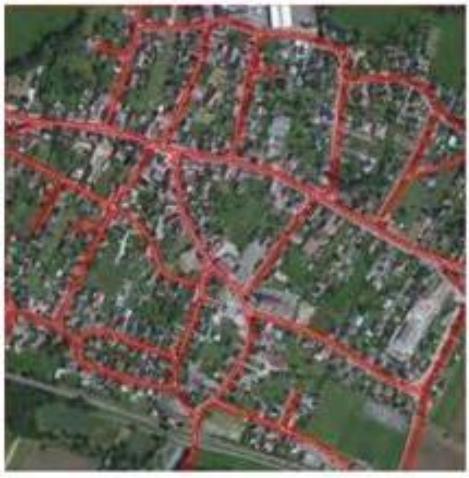

(a)

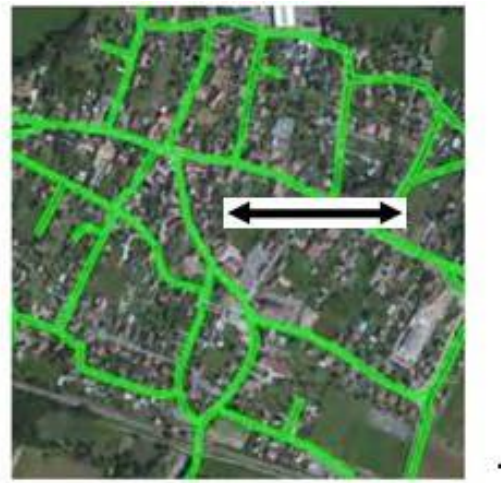

(b)

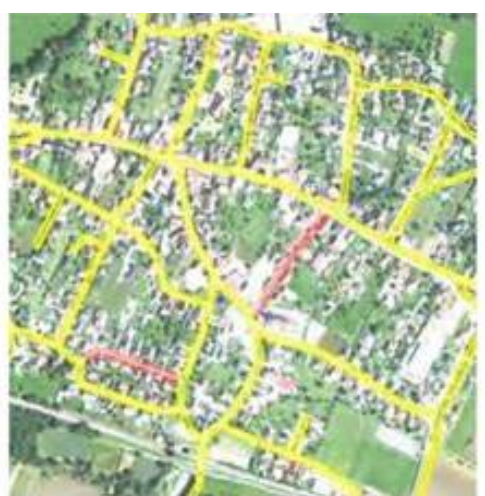

(c)

Figure 5. (a) Before disaster Road detection, (b) After disaster, (c) contacting two outputs.

Here, in figure 5.a and 5.b shows before after road segmentation and 5.c denotes the concatenation after overlapping both images. The yellow part is the commonly segmented or structured common roads after disaster and red refers the missing or destroyed road. More segmentations of roads are given in figure 3. The shortest path estimation algorithm will be implemented on the recent output from post disaster situation. It will extract the shortest path for sending relieves. The post disaster road images are manually collected from the road segmentation process. In this process we will combine both images for road detection using both kind of images, road map and satellite images. The image dataset for experiment was automatically retrieved from Road segmentation process. The intersection and the ending points of roads are orderly numbered. In this process, the intersection of roads at a position is separated into a set of intersection points, which depends on the number of road branches and they connect to other by small road segments.

The volcano and fire detect is marked based on the $\mathrm{L}^{*} \mathrm{a}^{*} \mathrm{~b}$ color space, and binary masking and it refers and marks the brightest part which is the creating point of volcano or fire. From this result shown in Figure 6, the perimeter of volcano's creating point can be measured.

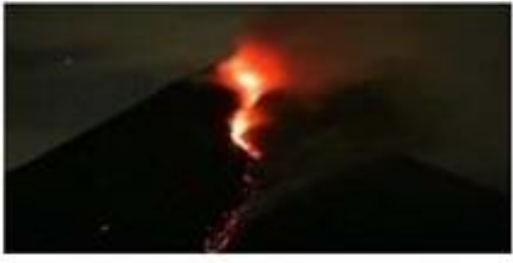

(a)

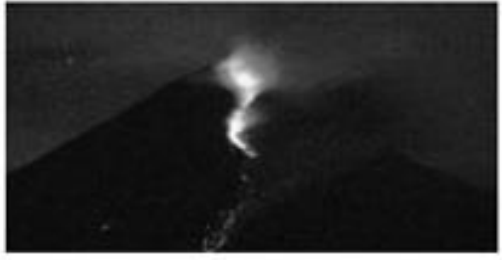

(b)

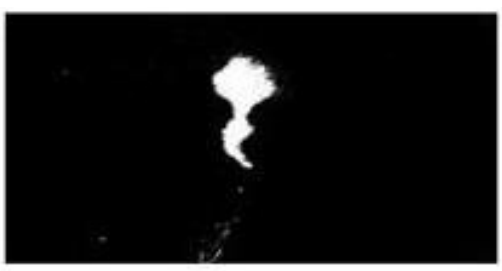

(c)

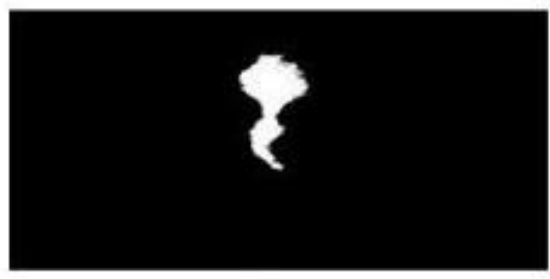

(d)

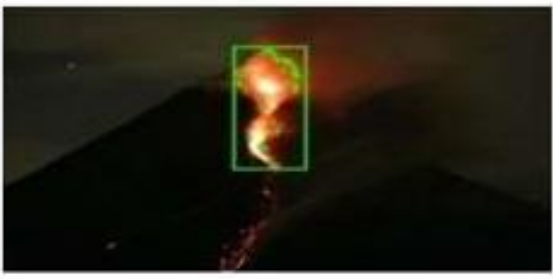

(e) 


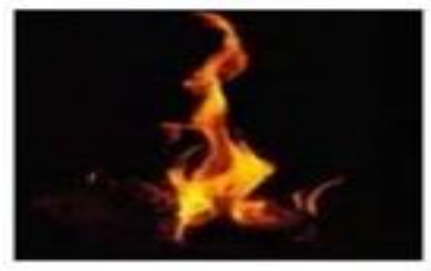

(a)

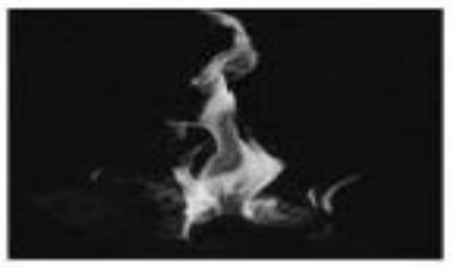

(b)

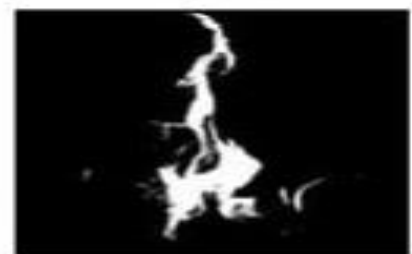

(c)

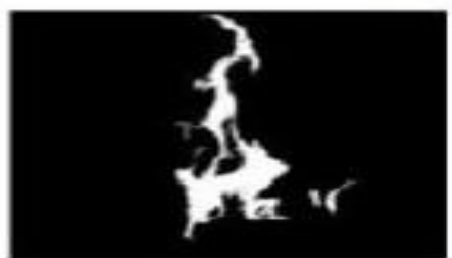

(d)

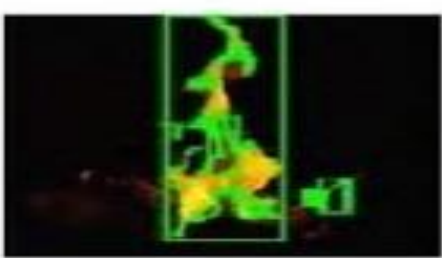

(e)

Figure 6. (a) segmented using k-mean cluster algorithm, (b) gray-scale conversion, (c) Binary Masking using Otsu Threshold algorithm, (d) Noise Cancelled and image reconstruction using flood fill, (e) Detected region

In our research we have some sample images different types of flood water dataset. When flood occurs, our system will compare flood affected area with our sample flood water dataset. Flood affected road images collected from satellite view are compared with the flood water dataset. If the water color of affected area matches with flood water dataset it will mark it with a particular color.

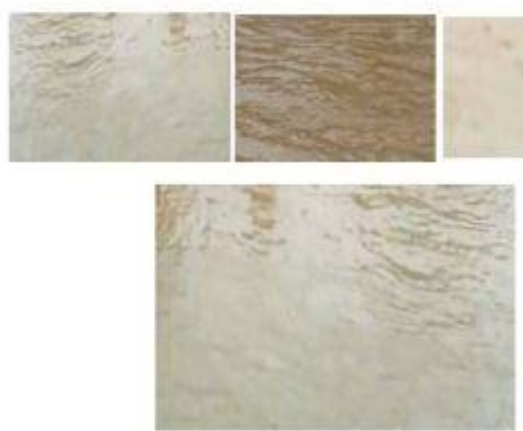

(a)

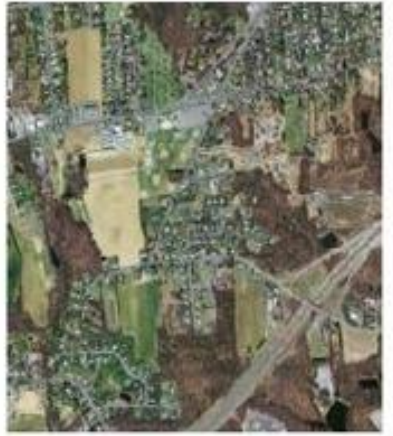

(b)

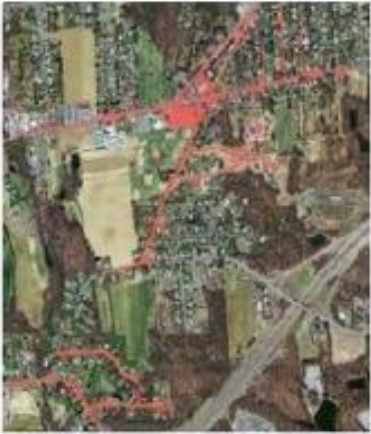

(c)

Figure 6. (a) Water texture, (b) primary image, (c) Marked detected region

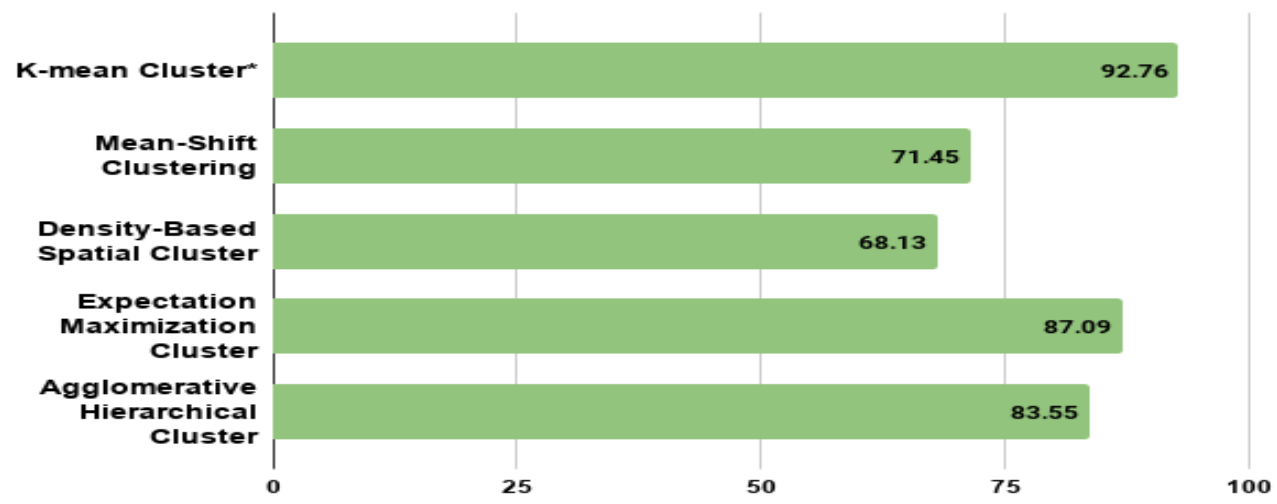

Figure 8. Comparison of clustering algorithms.

We compared the outcome of the clustering shown in Figure 8. with approached k-mean cluster and mean-shift, density-based spatial, expectation maximization, agglomerative hierarchical cluster algorithm. The outcomes showed that k-mean has the best outcomes and density-based spatial has the lowest. 
Another comparison is presented in Figure 9 to show affect of threshold value while converting in binary image comparing to masked image. The results indicates that, threshold value is 0.4 with $96.98 \%$ accuracy which is most suitable for binarization while road segmentation, and begin to decrease before and after that value.

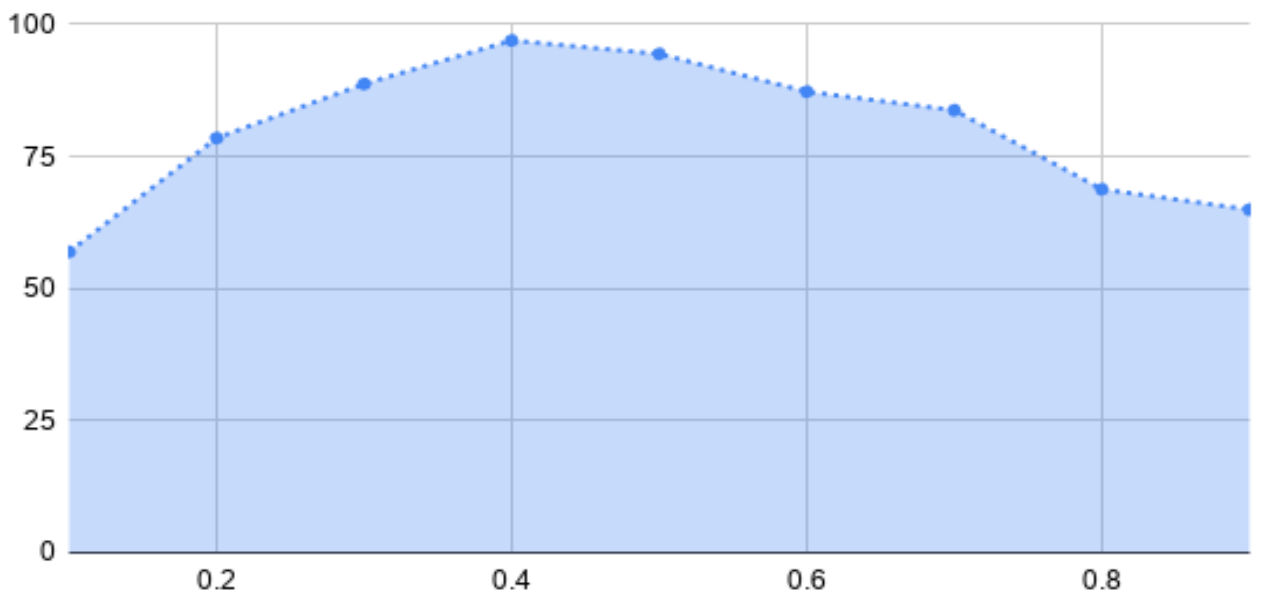

Figure 9. Threshold value vs accuracy

\section{CONCLUSION AND FUTURE WORK}

In this research we used different methods to identify all the available roads. We used K-means algorithm to compare the pre and post disaster road images. we used color-based segmentation method to detect fire and volcano hazards which can also detect flood affected area. we also applied shortest path estimation algorithm to find out the shortest road which is not blocked by any object. It will help us to reach the affected area as soon as possible. This research has come up with a novel approach to detect the shortest roads in disastrous moment so that our relief team easily find out a way to reach the affected area as soon as possible but there are still some complexities in our project. In future we will try to find out that complexities and find a solution for that. Moreover, we will make a graphical user interface (GUI) for this where all the methods will be merged and will be stored huge datasets and implement deep learning method [20] for better and feasible outcomes.

\section{REFERENCES}

[1] A. J. Cooner, Y. Shao, and J. B. Campbell, "Detection of Urban Damage Using Remote Sensing and Machine Learning Algorithms: Revisiting the 2010 Haiti Earthquake," Remote Sensing, vol. 8, no. 10, p. 868, 2016.

[2] S. W. Myint, M. Yuan, R. S. Cerveny, and C. P. Giri, "Comparison of Remote Sensing Image Processing Techniques to Identify Tornado Damage Areas from Landsat TM Data," Sensors, vol. 8, no. 2, pp. 1128-1156, 2008.

[3] A. Sumalan, D. Popescu and L. Ichim, "Flooded area detection using UAV images," 2016 24th Telecommunications Forum (TELFOR), Belgrade, 2016 , pp. 1-4.

[4] M. I. Pavel, S. M. Kamruzzaman, S. S. Hasan and S. R. Sabuj, "An IoT Based Plant Health Monitoring System Implementing Image Processing," 2019 IEEE 4th International Conference on Computer and Communication Systems (ICCCS), Singapore, 2019, pp. 299-303, doi: 10.1109/CCOMS.2019.8821782.

[5] Wei, P., Zhou, Z., Li, L., \& Jiang, J. (2018). Research on face feature extraction based on K-mean algorithm. EURASIP Journal on Image and Video Processing, 2018(1), 1-9.

[6] Modurodoluwa Adeyinka Okeowo, Hyongki Lee, Faisal Hossain, Augusto Getirana, "Automated Generation of Lakes and Reservoirs Water Elevation Changes From Satellite Radar Altimetry", Selected Topics in Applied Earth Observations and Remote Sensing IEEE Journal of, vol. 10, no. 8, pp. 3465-3481, 2017.

[7] F. U. Siddiqui and N. A. Mat Isa, "Enhanced moving K-means (EMKM) algorithm for image segmentation," in IEEE Transactions on Consumer Electronics, vol. 57, no. 2, pp. 833-841, May 2011.

[8] S. N. Sulaiman and N. A. Mat Isa, "Adaptive fuzzy-K-means clustering algorithm for image segmentation," IEEE Transactions on Consumer Electronics, vol. 56, pp. 2661-2668, 2010.

[9] D. Malyszko and S. T. Wierzchon, "Standard and Genetic k-means Clustering Techniques in Image Segmentation," 6th International Conference on Computer Information Systems and Industrial Management Applications (CISIM'07), Minneapolis, MN, 2007, pp. 299-304.

[10] Zhang, Y., Su, Y., Yang, J., Ponce, J., \& Kong, H. (2018). When Dijkstra meets vanishing point: a stereo vision approach for road detection. IEEE transactions on image processing, 27(5), 2176-2188.

[11] Milon, M. S. I., Pavel, M. I., Ehsan, M. S., Sadi, S. H., \& Sabuj, S. R. (2020). Application of Smart Appliance Using Internet of Things. In Innovations in Electronics and Communication Engineering (pp. 359-368). Springer, Singapore.

[12] R. I. Rumi, M. I. Pavel, E. Islam, M. B. Shakir and M. A. Hossain, "IoT Enabled Prescription Reading Smart Medicine Dispenser Implementing Maximally Stable Extremal Regions and OCR," 2019 Third International conference on I-SMAC (IoT in Social, Mobile, Analytics and Cloud) (I-SMAC), Palladam, India, 2019, pp. 134-138, doi: 10.1109/I-SMAC47947.2019.9032709.

[13] Pavel, M. I., Rumi, R. I., Fairooz, F., Jahan, S., \& Hossain, M. A. (2020, January). Deep Residual Learning Approach forPlant Disease Recognition. In International Conference on Mobile Computing and Sustainable Informatics (pp. 511-521). Springer, Cham. 


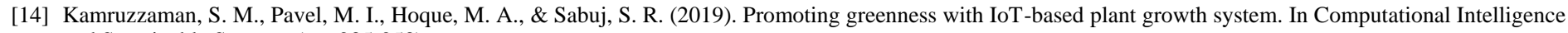
and Sustainable Systems (pp. 235-253).

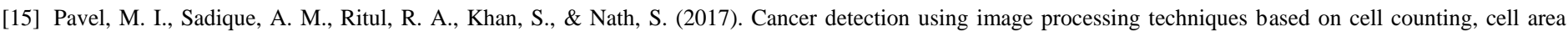
measurement and clump detection, BRAC Univeristy.

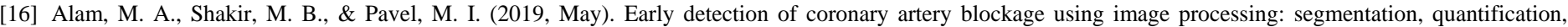

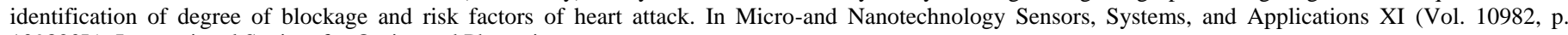
109820L). International Society for Optics and Photonics.

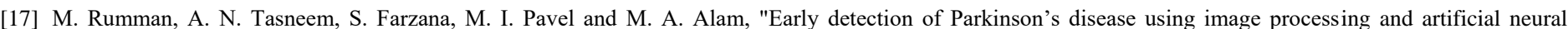

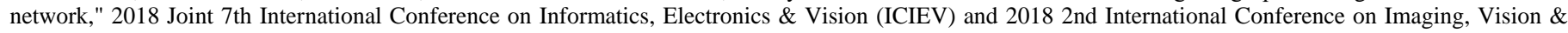
Pattern Recognition (icIVPR), Kitakyushu, Japan, 2018, pp. 256-261, doi: 10.1109/ICIEV.2018.8641081.

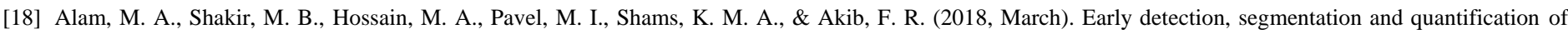

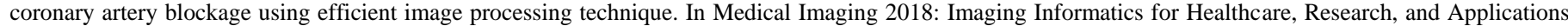
(Vol. 10579, p. 105791J). International Society for Optics and Photonics.

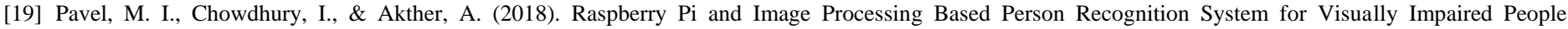
International Research Journal of Engineering and Technology, 5(7), 809-813.

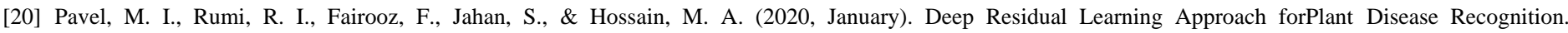
In International Conference on Mobile Computing and Sustainable Informatics (pp. 511-521). Springer, Cham.

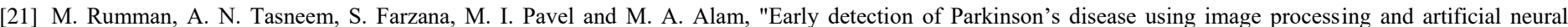

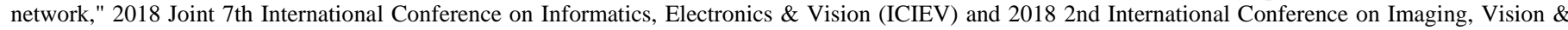
Pattern Recognition (icIVPR), Kitakyushu, Japan, 2018, pp. 256-261, doi: 10.1109/ICIEV.2018.8641081.

\section{AUTHORS}

(1)Mohadmmad Amzad Hossain, Department of Computer Science and Engineering, BRAC University, Email: mohammad.amzad.hossain@g.bracu.ac.bd

(2) Mohammad Tashauf Akand, , Department of Computer Science and Engineering, BRAC University, Email: tashauf.ananno@gmail.com

(3) Iftikharul Faridee Nur, Department of Computer Science and Engineering, American International University Bangladesh, Email: 17-34013-1@ student.aiub.edu

(4) Sazzatuz Zumma Ismam, Department of Electronics and Communications Engineering, East West University, Email: sazzatuzismam92@gmail.com

(5) MD. Fahim Shahriar Sakib, Department of Electrical and Electronic Engineering, BRAC University, Email: fahimshahriarsakib@gmail.com

(6) Tanvir Ahmed, Department of Electrical and Electronic Engineering, American International University Bangladesh, Email: tanvirahned@gmail.com

(7) Azmir Ahmed, Department of Electrical and Electronic Engineering, BRAC University, Email: azmirahmedf22@gmail.com

Correspondence Author - Sazzatuz Zumma Ismam, Department of Electronics and Communications Engineering, East West University, sazzatuzismam92 @gmail.com 